\title{
Adverse Drug Reaction Reports Regarding Abnormal Behavior After Oseltamivir Use in Children as Reported by Consumers or Healthcare Professionals
}

This article was published in the following Dove Press journal: Patient Preference and Adherence

\author{
Aoi Nodal-3 \\ Masami Tsuchiya (iD ${ }^{4,5}$ \\ Takamasa Sakai (iD) ${ }^{6}$ \\ Taku Obara iD ${ }^{1-3}$ \\ Nariyasu Mano ${ }^{3,4}$ \\ 'Division of Preventive Medicine and \\ Epidemiology, Tohoku University, Tohoku \\ Medical Megabank Organization, Sendai, \\ Miyagi, Japan; ${ }^{2}$ Tohoku University \\ Graduate School of Medicine, Sendai, \\ Miyagi, Japan; ${ }^{3}$ Department of \\ Pharmaceutical Sciences, Tohoku \\ University Hospital, Sendai, Miyagi, Japan; \\ ${ }^{4}$ Laboratory of Clinical Pharmacy, Tohoku \\ University Graduate School of \\ Pharmaceutical Sciences, Sendai, Miyagi, \\ Japan; ${ }^{5}$ Department of Pharmacy, Miyagi \\ Cancer Center, Natori, Miyagi, Japan; \\ ${ }^{6}$ Drug Informatics, Faculty of Pharmacy, \\ Meijo University, Nagoya, Aichi, Japan
}

Purpose: The purpose of this study was to assess the differences between consumer (patient) and healthcare professional submissions of adverse drug reaction (ADR) reports associated with certain antiviral treatments in children.

Material and Methods: We extracted ADR reports for children aged $<20$ years who received oseltamivir or similar drugs (zanamivir and amantadine) between April 2004 and May 2020 from the Japanese Adverse Drug Event Report database. Abnormal behavior after oseltamivir administration was reported frequently in the news in November 2005, and a Dear Healthcare Professional letter about abnormal behavior after oseltamivir use was issued on March 20, 2007. We compared the number of ADR reports by three periods: (1) before the news, (2) between the news and the letter, and (3) after the letter. These reports were tabulated and analyzed after stratification according to the reporter (healthcare professionals only, patients and healthcare professionals, patients only), patient age $(<10$ years, 10-19 years), and ADR (abnormal behavior, other ADRs).

Results: For the reports from healthcare professionals only, the number of reports per quarter associated with oseltamivir was largest during the period between the news about abnormal behavior after oseltamivir use and publication of the Dear Healthcare Professional letter. The reports from patients only about abnormal behavior after oseltamivir use were first reported after publication of the letter. The proportions of reports from patients only about abnormal behavior with oseltamivir were $81.0 \%$ and $92.2 \%$ for ages $<10$ and $10-19$ years, respectively. A ripple effect of increasing reports was observed with zanamivir or amantadine.

Conclusion: Reports from patients only might increase in response to the media more than reports from healthcare professionals only or patients and healthcare professionals do. The ADR reports from patients must be carefully assessed from the perspective of when they were reported.

Keywords: children, oseltamivir, drug safety, spontaneous reporting system, pharmacovigilance

\section{Introduction}

There is a lack of safety and efficacy information about the use of drugs in children. Key reasons include the wide range of patients - from newborns to adolescentscovered by pediatric drug development and the need for detailed attention to dosage forms and pharmacokinetics in this population. Furthermore, such drug development requires consent from pediatric patients in addition to consent from their parents or guardians to conduct clinical trials. ${ }^{1}$ In fact, according to a previous
Division of Preventive Medicine and Epidemiology, Tohoku University, Tohoku Medical Megabank Organization, 2-I

Seiryou-Cho, Aoba-Ku, Sendai, 980-8573, Japan

Tel +8I 227178104

$\mathrm{Fax}+81227178106$

Email obara-t@hosp.tohoku.ac.jp
Patient Preference and Adherence 202I:15 533-54I 
survey targeting children who had visited a hospital, about $70 \%$ of drugs prescribed for children in Japan were reportedly used off label. ${ }^{2}$ Different approaches to exploring drug safety, including registries and a careful review of the literature, are vital. ${ }^{3}$

The collection of adverse drug reaction (ADR) reports began in 1967 in Japan. Companies and healthcare professionals report ADRs directly to the Pharmaceutical and Medical Devices Agency (PMDA), which was established by the Ministry of Health, Labour and Welfare. According to the Pharmaceutical and Medical Device Act, the PMDA recommends that pharmaceutical companies and healthcare professionals report ADRs even if the causal relationship between drug use and an ADR is unclear. Since 2012, the PMDA has made parts of ADR reports available to the public through the Japanese Adverse Drug Event Report (JADER) database. We previously reported that the most frequently reported drug used in children in the JADER database was oseltamivir. ${ }^{5}$ A Dear Healthcare Professional letter about abnormal behavior after oseltamivir use in children aged $>10$ years was issued on March $20,2007 .{ }^{4}$ We demonstrated that administrative responses to safety information might be determinants of increasing ADR reports. ${ }^{5,6}$

Patients are less likely than healthcare professionals to be affected by preconceived notions about ADRs, so patient reports have the potential to identify new ADRs that have not been reported by healthcare professionals. ${ }^{7-9}$ Patient reporting systems already exist in many countries. For patients in Japan, the Direct Patient Reporting System for ADR, in which patients and consumers report ADRs directly to the PMDA, was tentatively started in 2012 as a pilot program, and full-scale operation of the system began on March 26, 2019. ${ }^{10,11}$ However, the bulk of reports about Japanese children submitted by patients has not yet been fully assessed. Because the number of reports from the Direct Patient Reporting System for ADR is small, we used the JADER database to assess the differences between ADR reports from patients and those from healthcare professionals.

\section{Material and Methods}

\section{Data Source}

ADR reports included in the JADER database from April 1, 2004, to May 31, 2020, were downloaded in September 2020 in the accordance with relevant data protection and privacy regulations (https:/www.pmda.go.jp/ $\underline{\text { safety/info-services/drugs/adr-info/suspected-adr/0003. }}$ html).

The JADER database consists of four tables: (1) patient demographic information, (2) drug information, (3) adverse events, and (4) primary diseases. The adverse reaction and underlying disease fields in the database are described by Preferred Terms (PTs) in the Japanese version of the Medical Dictionary for Regulatory Activities $\left(\operatorname{MedDRA}^{\circledR} / \mathrm{J}\right)$. We used $\operatorname{MedDRA}^{\circledR} / \mathrm{J}$ version 23.1 (International Council for Harmonisation of Technical Requirements for Pharmaceuticals for Human Use, Geneva, Switzerland) in this study. Collected information included patient details (age and sex), the type of report sender (pharmaceutical company or healthcare facility), the qualification of the reporter (doctor, pharmacist, healthcare professional, consumer, or lawyer), the reporting year and quarter, the suspected drug, and the ADR coded according to PTs. With regard to reporter qualifications, multiple professions (eg, physician and pharmacist, physician and healthcare professional) could be listed as a reporter for a single ADR report. Because consumers cannot report ADRs directly through the Drug and Medical Device Safety Information Reporting System, consumers report their suspected ADRs to pharmaceutical companies or post them to social media. Companies collect this information and submit it as reports from consumers, so reports from consumers only were almost always reported as company reports. We defined consumers in the JADER database as patients in this study.

\section{Statistical Analysis}

The spontaneous reports were extracted from the JADER dataset. We categorized the reports into three categories by reporter type: (1) healthcare professionals only, (2) patients and healthcare professionals, and (3) patients only. Healthcare professionals included physicians, dentists, pharmacists, and healthcare professionals listed in the "Qualifications of the Reporter" section in the JADER database. We defined the patients without healthcare professionals category as patients only. If both patients and healthcare professionals were listed as a reporter for a single ADR report, we defined the reporter type as patients and healthcare professionals. We evaluated the ADR reports about use of oseltamivir or similar drugs (zanamivir and amantadine). Abnormal behavior after oseltamivir administration was reported frequently in the news in November 2005, and a Dear Healthcare Professional letter about abnormal behavior during 
oseltamivir use in children aged 10-19 years was issued on March 20, 2007. We compared ADR reports by three periods as follows: (1) before the news, (2) between the news and the letter, and (3) after the letter. Because the JADER database does not included detailed reporting dates, only quarterly aggregation is possible. Since altered state of consciousness, abnormal behavior, delirium, hallucinations, delusions, and seizures were added to the revised package insert for oseltamivir in May 2004, we defined this description as an abnormal behavior and fear, agitation, amnesia, hallucination (visual), hallucination (auditory), intentional self-injury, aggression, illusion, confusional state, suicide attempt, completed suicide, and restlessness as abnormal behavior were also defined as related by the PTs in this study.

The reports were tabulated and analyzed after stratification according to the reporter (healthcare professionals only, patients and healthcare professionals, patients only), patient age ( $<10$ years, $10-19$ years), and ADR (abnormal behavior, other ADRs). Since the JADER only included age information as a categorical variable, we extracted ADR reports for children aged $<10$ and $10-19$ years.
Ethical approval for the study was obtained from the Institutional Review Board of Tohoku University School of Medicine (2017-1-506).

\section{Results}

A total of 646,779 ADR reports were downloaded from the PMDA website in September 2020. Of these, the number of spontaneous reports was 505,465 (78.2\%). We extracted 33,338 ADR reports (5.2\%) for children aged $<10$ and $10-19$ years. After extraction of ADR reports that included oseltamivir $(\mathrm{n}=893)$, we excluded unknown reporter reports $(n=4)$. A total of 889 reports $(0.14 \%$ of the initial total) were finally included in this analysis. Overall, 835 reports were from healthcare professionals only, 19 were from patients and healthcare professionals, and 35 were from patients only (Figure 1). With regard to zanamivir and amantadine, a total of 679 reports $(0.11 \%$ of the initial total) were finally included in this analysis. Of these 679,565 reports were from healthcare professionals only, 66 were from patients and healthcare professionals, and 48 were from patients only (Figure 1). The total number of reports increased year by year; among children

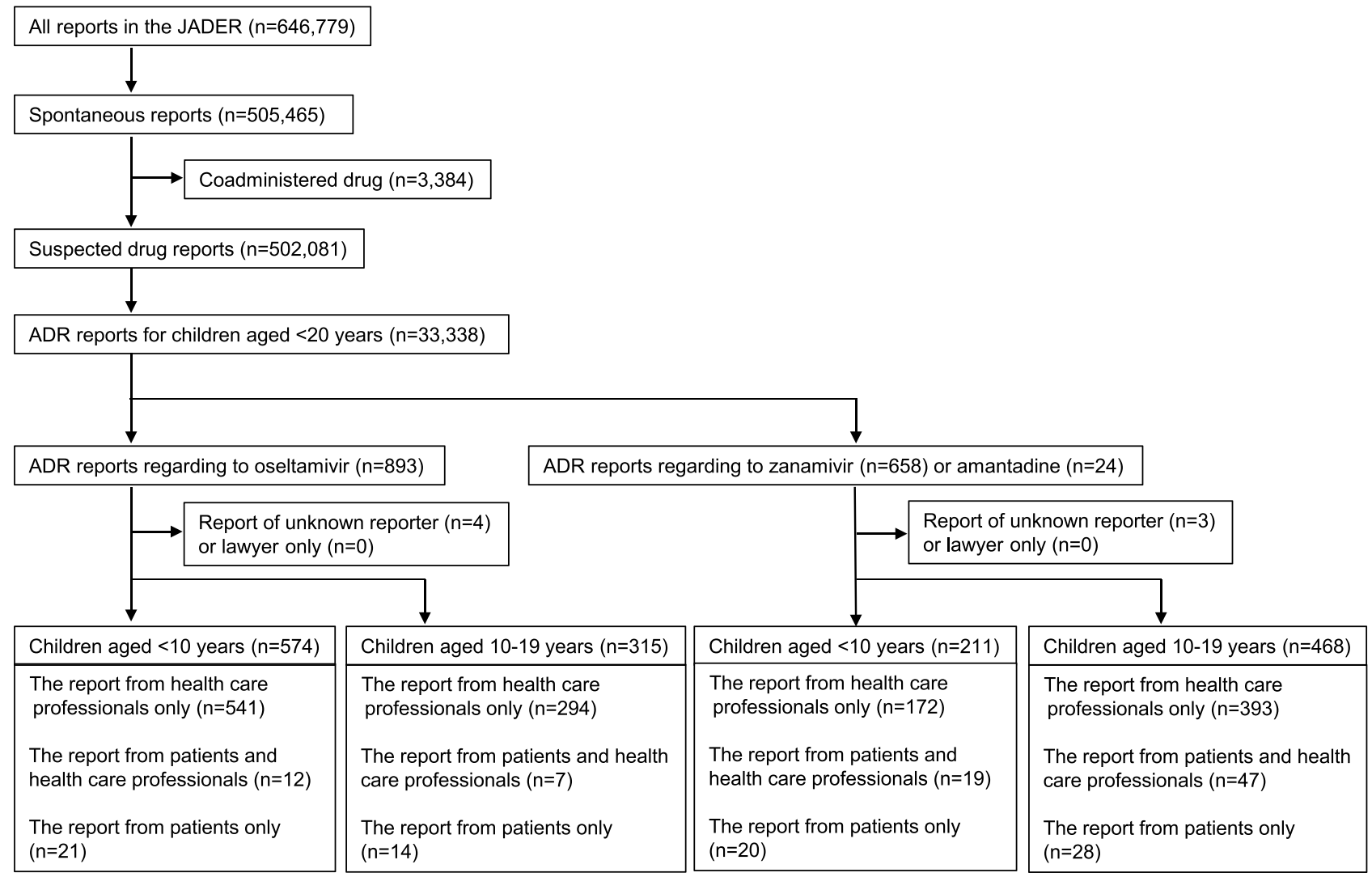

Figure I Selection of the analysis set and exclusion criteria. Abbreviation: ADR, adverse drug reaction. 
aged $<20$ years, ADR reports about oseltamivir increased from 2005 to 2008 and especially in 2007, and about zanamivir increased from 2007 to 2009, especially in 2009 (Figure 2).

When we looked at the reports from healthcare professionals only without stratifying by age, the number of reports per quarter associated with oseltamivir was the largest between the news release and publication of the letter. The reports from patients only about abnormal behavior after the use of oseltamivir were first reported after publication of the letter (Table 1). The proportions of reports about abnormal behavior after oseltamivir use from those submitted by healthcare professionals only, healthcare professionals and patients, and patients only were $71.4 \%, 66.7 \%$, and $92.9 \%$, respectively, for patients aged $10-19$ years; these proportions were $67.3 \%, 75.0 \%$, and $81.0 \%$, respectively, for patients aged $<10$ years. The proportion of the reports from patients only about abnormal behavior after oseltamivir use was $4.9 \%$.

The proportion of the reports associated with abnormal behavior after zanamivir and amantadine use from patients only was $85.7 \%$ for patients aged $10-19$ years, and it was $90.0 \%$ for patients aged $<10$ years. The number of reports increased after the publication of the letter about oseltamivir (Table 2). The proportion of reports from patients only about abnormal behavior after zanamivir or amantadine was $8.4 \%$.

\section{Discussion}

Although the total number of reports increased year by year; among children aged $<20$ years, many ADR reports from 2005 to 2008, and especially in 2007, were about oseltamivir. The period with the highest frequency of oseltamivir-related ADRs differed between patient and healthcare professional reporting groups. For the reports from healthcare professionals only or combined with patients, the number of reports per quarter of abnormal behavior after oseltamivir use was the largest during the period between the news about abnormal behavior with oseltamivir use and publication of the Dear Healthcare Professional letter. This period might reflect healthcare professional attempts to collect ADRs carefully and verify a causal relationship between oseltamivir and abnormal behavior. For the reports from patients only, the number of reports about abnormal behavior after oseltamivir use increased after the publication of the letter. Patients might have responded because of the news report but reported these ADRs later, during $2007 \mathrm{flu}$ season. That is, reports from patients only might reflect a time lag from the news release to the submission of ADR reports. The number of reports from patients and healthcare professionals, unlike those from patients only, did not increase after the letter. The involvement of healthcare professionals in patient reporting might reduce the influence of the media on patients. Moreover, among the reports about oseltamivir from patients only, the percentage of reports of abnormal behavior was $92.9 \%$ for those aged $10-19$ years and was $81.0 \%$ for those $<10$ years - both of which were higher than the reports from healthcare professionals or patients and healthcare professionals. The reporting of abnormal behavior as an ADR associated with the use of these drugs was quite high; conversely, the reporting rate of other ADRs was relatively low, so it might be difficult to detect unknown ADRs caused by oseltamivir using disproportionality analysis.

After the news about abnormal behavior associated with oseltamivir use, a drug safety letter was published by a Japanese regulatory agency on March 20, 2007. Although the causal relationship between oseltamivir and abnormal behavior has not been clarified, the revision of the package insert in 2007 stated that oseltamivir is contraindicated in children aged $>10$ years. As a result, few reports about patients aged 10-19 years were reported before the contraindications were lifted in August 2018. During the time after the publication of the safety letter, zanamivir was often used as an alternative to oseltamivir. ${ }^{12}$ The reports from patients only about abnormal behavior after the use of zanamivir or amantadine were also first reported after publication of the safety letter for oseltamivir, and the proportion of reports from patients only about abnormal behavior after use of zanamivir or amantadine was higher than reports after use of oseltamivir. The ripple effect of the publication of the safety letter for oseltamivir that caused more reporting about zanamivir or amantadine might be stronger on the reporting behavior of patients than that of healthcare professionals.

It became clear that patients might be more affected than healthcare professionals (alone or combined with patients) by the media in this study. Because ADR reports from patients are affected by various information and administrative responses, we assessed the ADR results with regard to the time of the report.

Although ADR reports from consumers within the JADER database did not completely match reports of patients in the overseas database, we assessed the differences in ADR reports by three reporter types. In Japan, the 
A 60000

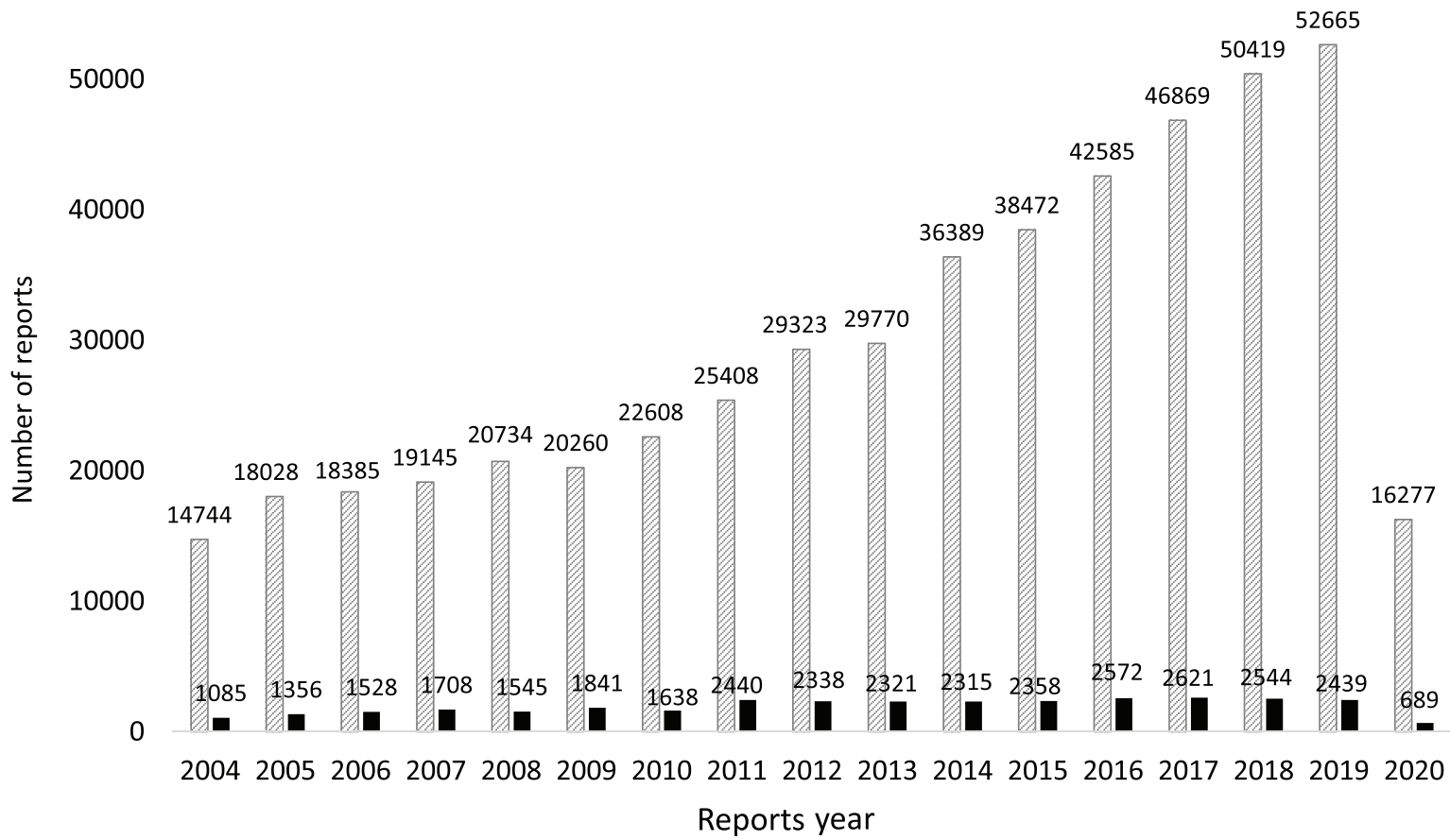

Total number of spontaneous reports in the JADER - Total number of ADR reports in children aged $<20$ years

B

400

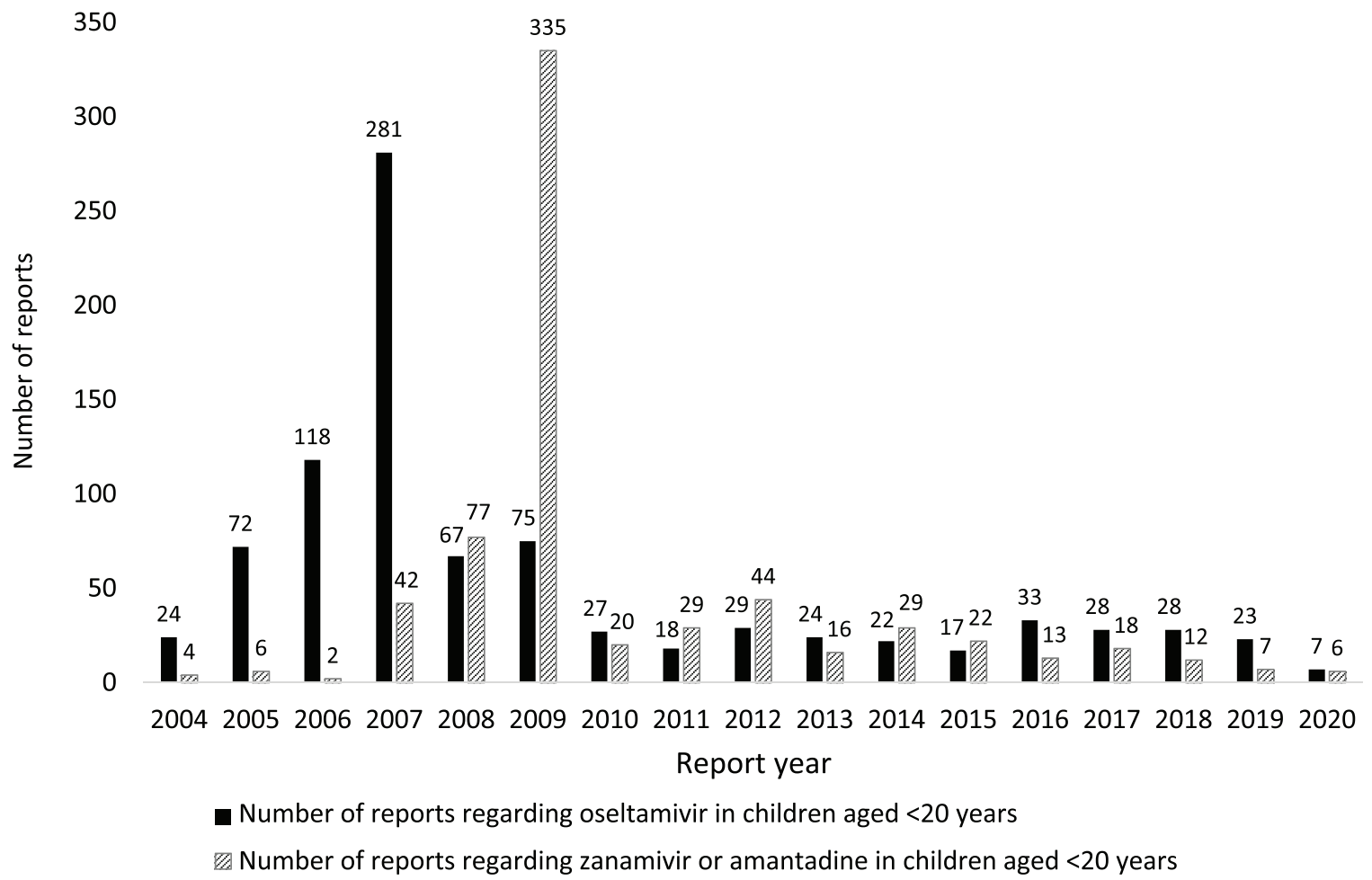

Figure 2 (A) Total number of spontaneous reports in the JADER. (B) Total number of ADR reports in children aged $<20$ years. 


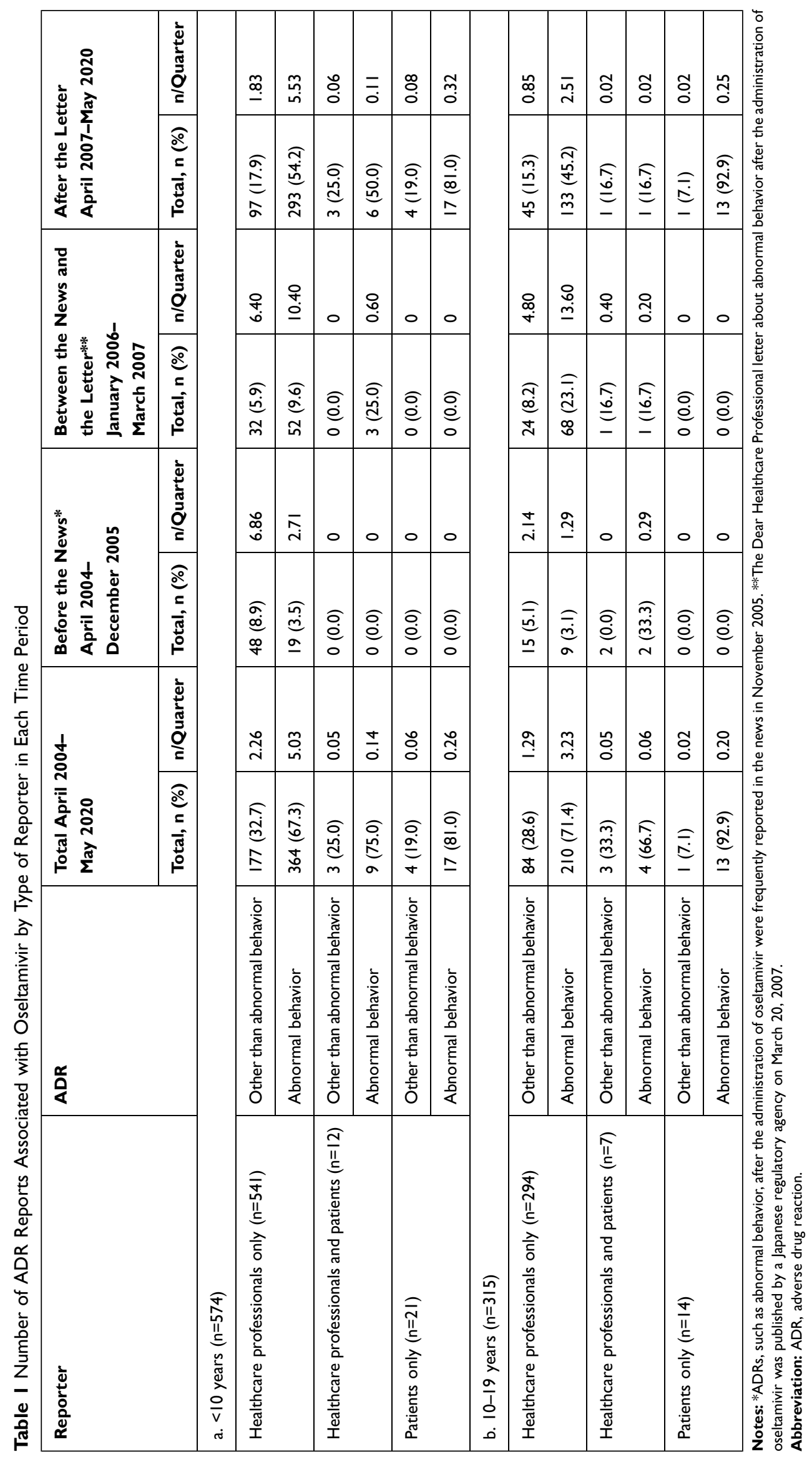




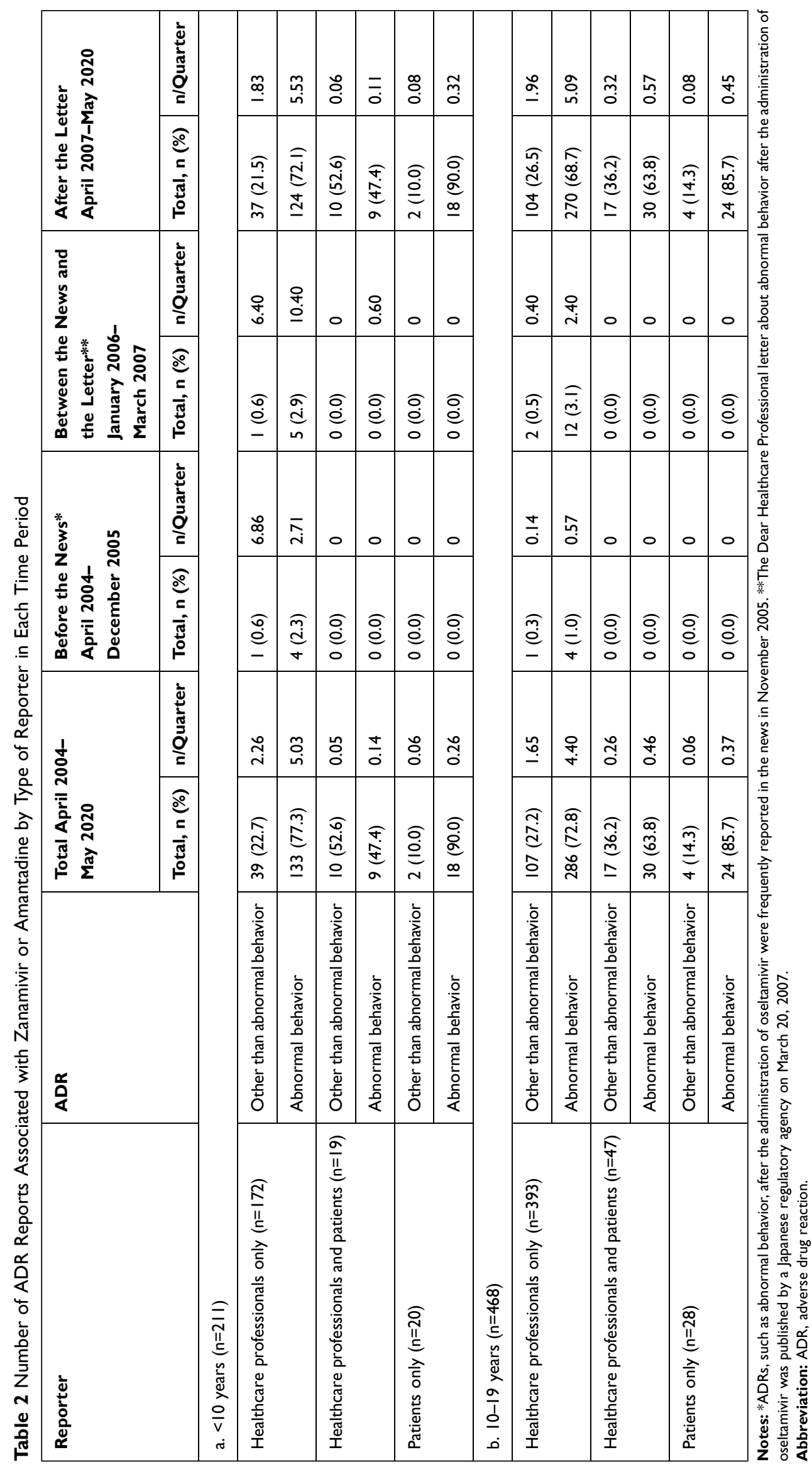


Direct Patient Reporting System for ADR, in which patients and consumers can report ADRs directly to the PMDA, began on March 26, 2019. However, the JADER database has not included the reports from this system. From the implementation of the system to March 2020, in database of the Direct Patient Reporting System for ADR, there were only three and eight reports for children aged $<10$ years and 10-19 years, respectively. ${ }^{13}$ As often seen in other countries, many patients are not aware of the patient reporting system, so it is necessary to educate patients about the patient reporting system itself. ${ }^{14}$ In Denmark, a leaflet was distributed to pharmacies and general practitioners in 2007, in which patients were encouraged to electronically report ADRs potentially caused by their medicines. ${ }^{15}$ As shown in studies about patient reporting in foreign countries, ${ }^{16,17}$ more promotion of the patient reporting system is needed to collect and accumulate drug safety information for use in children and to evaluate the trends and developments in ADR reports collected while considering the influence of the media and administrative responses.

The JADER database is a spontaneous ADR reporting database, and it has multiple limitations, such as the reporting of temporal associations, the unconfirmed diagnoses, and a lack of denominator of the user, and an unbiased comparison of groups such as healthy individuals taking drugs. ${ }^{18}$ Because of these limitations, clarifying causality between drugs and ADRs from the JADER database could be difficult. Moreover, because the JADER database does not included detailed reporting dates, only quarterly aggregation is possible. Also, it is not possible to analyze the situation according to World Health Organization age group classification, ${ }^{19}$ because the JADER database only includes age information as a categorical variable (eg, children aged $<10$ years and 10-19 years). However, the database enables the early detection of signals. $^{20}$

\section{Conclusion}

Reports from patients only might increase in response to the media more than reports from healthcare professionals only and patients and healthcare professionals do. The ADR reports from patients must be carefully assessed from the perspective of when they were reported.

\section{Abbreviations}

ADR, adverse drug reaction; JADER, Japanese Adverse Drug Event Report; MedDRA ${ }^{\circledR} / J$, the Japanese version of the Medical Dictionary for Regulatory Activities; PMDA, Pharmaceutical and Medical Devices Agency; PT, preferred term.

\section{Acknowledgments}

This research was supported by the Research on Regulatory Harmonization and Evaluation of Pharmaceuticals, Medical Devices, Regenerative and Cellular Therapy Products, Gene Therapy Products, Cosmetics from the Japan Agency for Medical Research and Development (20mk0101143h0002), and the Japan Society for the Promotion of Science (JSPS) (19K07213). We would like to thank The Charlesworth Author Services for English language editing.

\section{Disclosure}

The authors report no conflicts of interest in this work.

\section{References}

1. Ministry of Health and Welfare. Notification no.1334: clinical investigation of medicinal products in the pediatric population. Available from: https://www.pmda.go.jp/files/000156072.pdf. Accessed November 5, 2020. Japanese.

2. Morita S. The report of health and labour sciences research grants; 2011. Available from: https://mhlw-grants.niph.go.jp/niph/search/NIDD00.do? resrchNum=199900758A. Accessed November 5, 2020. Japanese.

3. Rieder M. Adverse drug reactions across the age continuum: epidemiology, diagnostic challenges, prevention, and treatments. $J$ Clin Pharmacol. 2018;58(Suppl 10):S36-S47. doi:10.1002/jcph.1115

4. Ministry of Health, Labour and Welfare. The dear healthcare professional letter. Abnormal behavior after tamiflu use. [cited March 20, 2007]. Available from: https://www.pmda.go.jp/files/000147877.pdf. Accessed November 5, 2020. Japanese.

5. Noda A, Sakai T, Obara T, et al. Characteristics of pediatric adverse drug reaction reports in the Japanese adverse drug event report database. BMC Pharmacol Toxicol. 2020;21(1):36. doi:10.1186/ s40360-020-00412-7

6. Noda A, Sakai T, Tsuchiya M, Oyanagi G, Obara T, Mano N. Characteristics of adverse events following immunization reporting in children: the Japanese adverse drug event report database. Vaccines. 2020;8(3):E357. doi:10.3390/vaccines 8030357

7. Blenkinsopp A, Wilkie P, Wang M, Routledge PA. Patient reporting of suspected adverse drug reactions: a review of published literature and international experience. Br J Clin Pharmacol. 2007;63 (2):148-156. doi:10.1111/j.1365-2125.2006.02746.x

8. van Hunsel F, Talsma A, van Puijenbroek E, de Jong-van den Berg L, van Grootheest K. The proportion of patient reports of suspected ADRs to signal detection in the Netherlands: case-control study. Pharmacoepidemiol Drug Saf. 2011;20(3):286-291. doi:10.1002/ pds.2092

9. Inácio P, Cavaco A, Airaksinen M. The value of patient reporting to the pharmacovigilance system: a systematic review. $\mathrm{Br} J$ Clin Pharmacol. 2017;83(2):227-246. doi:10.1111/bcp.13098

10. Yamamoto M, Kubota K, Okazaki M, et al. Patients views and experiences in online reporting adverse drug reactions: findings of a national pilot study in Japan. Patient Prefer Adherence. 2015; 9:173-184. doi:10.2147/PPA.S75761 
11. Ministry of Health, Labour and Welfare. Direct patient reporting of adverse drug reactions procedures for implementation. [cited March 26, 2019]. Available from: https://www.pmda.go.jp/files/000228627. pdf. Accessed November 5, 2020. Japanese.

12. The Pharmaceuticals and Medical Devices Agency. A trial study report on the actual situation of prescriptions and the effects of safety measures using receipt data. Available from: https://www.pmda.go.jp/ files/000147697.pdf. Accessed November 5, 2020. Japanese.

13. The Pharmaceuticals and Medical Devices Agency. Information on reported ADR reports. Available from: https://www.pmda.go.jp/safety/ reports/patients/0002.html. Accessed November 5, 2020. Japanese.

14. Al Dweik R, Stacey D, Kohen D, Yaya S. Factors affecting patient reporting of adverse drug reactions: a systematic review. $\mathrm{Br} J \mathrm{Clin}$ Pharmacol. 2017;83(4):875-883. doi:10.1111/bcp.13159

15. Danish Health Agency. Adverse drug reaction reported by consumers in Denmark - compared with reports from healthcare professionals. Available from: https://laegemiddelstyrelsen.dk/ /media/ B71CB7AF2879471ABE9DCF23BF853B18.ashx. Accessed November $5,2020$.

16. McLernon DJ, Bond CM, Hannaford PC, et al. For yellow card collaboration. Adverse drug reaction reporting in the UK: a retrospective observational comparison of yellow card reports submitted by patients and healthcare professionals. Drug Saf. 2010;33 (9):775-788. doi:10.2165/11536510-000000000-00000
17. Dweik RA, Yaya S, Stacey D, Kohen D. Spontaneous adverse drug reaction reporting by patients in Canada: a multi-method study-study protocol. Springerplus. 2016;5(1):213. doi:10.1186/s40064-016-18 38-9

18. Zhou W, Pool V, Iskander JK, et al. Surveillance for safety after immunization: vaccine adverse event reporting system (VAERS) United States, 1991-2001. MMWR Surveill Summ. 2003;52(1):1-24.

19. World Health Organization. Paediatric age categories to be used in differentiating between listing on a model essential medicines list for children. Available from: http://archives.who.int/eml/expcom/chil dren/Items/PositionPaperAgeGroups.pdf. Accessed November 4, 2020.

20. Sakai T, Ohtsu F, Mori C, Tanabe K, Goto N. Signal of miscarriage with aripiprazole: a disproportionality analysis of the Japanese adverse drug event report database. Drug Saf. 2017;40(11):11 41-1146. doi:10.1007/s40264-017-0560-z
Patient Preference and Adherence

\section{Publish your work in this journal}

Patient Preference and Adherence is an international, peer-reviewed, open access journal that focusing on the growing importance of patient preference and adherence throughout the therapeutic continuum. Patient satisfaction, acceptability, quality of life, compliance, persistence and their role in developing new therapeutic modalities and compounds to optimize clinical outcomes for existing disease states are major areas of interest for the journal. This journal has been accepted for indexing on PubMed Central. The manuscript management system is completely online and includes a very quick and fair peer-review system, which is all easy to use. Visit http:// www.dovepress.com/testimonials.php to read real quotes from published authors. 